the objects' last 27 orbits around each other, compared with just 5 or so from the first detection. This enabled the researchers to get a test of general relativity that was in some respects twice as precise as their test during the first detection.

This was true even though the September event was 'louder' than the December event. The first two black holes weighed as much as 36 and 29 times the mass of the Sun, respectively, whereas the holes in the second pair were relatively lightweight, at 14 and 8 solar masses, and radiated one-third as much gravitational energy. (In both cases, the black-hole pairs might have been orbiting each other for millions or billions of years, but LIGO captured only the finale, when the orbits and the gravitational waves that they produced had frequencies within the observatory's window of sensitivity.)

\section{WELL-TIMED GIFT}

The LIGO and Virgo scientists estimate that both collisions occurred more than 400 megaparsecs (1.3 billion light years) from Earth, although the distances could not be measured precisely. The teams presented their latest results on 15 June at a meeting of the American Astronomical Society in San Diego, California, and published them in Physical Review Letters (B. P. Abbott et al. Phys. Rev. Lett. 116, 241103; 2016).

The latest discovery was especially exciting for physicist Chad Hanna, a LIGO collaborator at Pennsylvania State University in University Park. When Hanna got the alert by text message, it was still Christmas Day in the United States, and he was with family. The collaboration requires members to keep data completely confidential, so Hanna hopped out of his chair, got his laptop and went upstairs to an empty room. At first, he was sceptical, he says: "I just didn't think the Universe had such a sense of humour to send a real event on Christmas."

But he soon realized that although the signal was relatively quiet, it was genuine. It was a crucial test for the software that combs the data from the two observatories in real time, which he helped to design. The system could catch events even if they were half-buried in noise, but didn't produce too many false positives.

\section{RESET}

Ultimately, the automated alerts from LIGO will notify dozens of teams of astronomers as well. The researchers will then reposition their telescopes in the hope of detecting visible light, or other electromagnetic waves, that originate from the same events that produce the gravitational waves.

After its first four-month science run between September 2015 and January 2016, the US\$620-million Advanced LIGO has shut down for an upgrade. It is scheduled to start another run this September, accompanied by an upgraded Virgo.

The results released last week complete the search for black-hole mergers in LIGO's autumn run, but the collaboration is still sifting through its data for other types of event - and may yet announce further discoveries even before the next run begins. In particular, the international Einstein@Home project is looking for signals with the help of computers belonging to volunteers around the world.

\section{CORRECTIONS}

It costs the United Kingdom $£ 161$ million per week to be in the EU, not $£ 250$ million, as stated in the Editorial 'Turning point' (Nature 534, 295; 2016). The News story 'Gene therapies pose million-dollar conundrum' (Nature 534, 305-306; 2016) should have said that cancer drugs that unleash the power of the immune system cost up to $\$ 40,000$ per month, not per year. And the News Feature 'The secret history of ancient toilets' (Nature 533, 456-458; 2016) incorrectly said that roundworms and whipworms cause dysentery - they cause problems such as malnutrition. 